\title{
Predictive Model for Ice Formation on Superhydrophobic Surfaces
}

\section{Citation}

Bahadur, Vaibhav, Lidiya Mishchenko, Benjamin Hatton, J. Ashley Taylor, Joanna Aizenberg, and Tom Krupenkin. 2011. "Predictive Model for Ice Formation on Superhydrophobic Surfaces." Langmuir 27 (23) (December 6): 14143-14150. doi:10.1021/la200816f.

\section{Published Version}

doi:10.1021/la200816f

\section{Permanent link}

http://nrs.harvard.edu/urn-3:HUL.InstRepos:37256172

\section{Terms of Use}

This article was downloaded from Harvard University's DASH repository, and is made available under the terms and conditions applicable to Open Access Policy Articles, as set forth at http:// nrs.harvard.edu/urn-3:HUL.InstRepos:dash.current.terms-of-use\#OAP

\section{Share Your Story}

The Harvard community has made this article openly available.

Please share how this access benefits you. Submit a story.

\section{Accessibility}




\title{
Predictive model for ice formation on superhydrophobic
}

\section{surfaces}

\author{
Vaibhav Bahadur', Lidiya Mishchenko ${ }^{2,3}$, Benjamin Hatton ${ }^{2,3}$, J. Ashley Taylor ${ }^{1}$, Joanna Aizenberg ${ }^{2,3,4}$, \\ Tom Krupenkin*1
}

${ }^{1}$ Department of Mechanical Engineering, University of Wisconsin-Madison, 1513 University Avenue, Madison, WI 53706

${ }^{2}$ School of Engineering and Applied Sciences, Harvard University, Cambridge, MA 02138

${ }^{3}$ Wyss Institute for Biologically Inspired Engineering, Harvard University, Cambridge, MA 02138

${ }^{4}$ Kavli Institute for Bionano Science \& Technology, Harvard University, Cambridge, MA 02138

*Corresponding author: tnk@engr.wisc.edu

\begin{abstract}
Prevention and control of ice accumulation has important applications in aviation, building construction and energy conversion devices. One area of active research concerns the use of superhydrophobic surfaces for preventing ice formation. The present work develops a physics-based modeling framework to predict ice formation on cooled superhydrophobic surfaces resulting from impact of supercooled water droplets. This modeling approach analyzes the multiple phenomena influencing ice formation on superhydrophobic surfaces through the development of submodels describing droplet impact dynamics, heat transfer and heterogeneous ice nucleation. These models are then integrated together to achieve a comprehensive understanding of ice formation upon impact of liquid droplets at freezing conditions. The accuracy of this model is validated by its successful prediction of the experimental findings which demonstrate that superhydrophobic surfaces can fully prevent freezing of impacting water droplets down to surface temperatures as low as -20 to $-25{ }^{\circ} \mathrm{C}$. The model can be used to study the influence of surface morphology, surface chemistry, and fluid and thermal properties on dynamic ice formation, and identify parameters critical to achieving ice-phobic surfaces. The framework in the present work is the
\end{abstract}


first detailed modeling tool developed for the design and analysis of surfaces for various ice prevention/reduction strategies.

\section{Introduction}

Ice formation adversely affects many aspects of everyday living through frozen windshields, downed power lines and burst pipes. Beyond these commonplace negative impacts, the operation of aircrafts $^{1}$ and wind turbines ${ }^{2}$ is severely impacted during icing conditions due to safety and energy efficiency considerations. The commonly used active ice removal strategies have big drawbacks; heating consumes energy while the environmental footprint associated with freeze retarding chemicals limits their use as deicing agents.

A survey of recent literature indicates growing interest in the use of passive techniques for ice mitigation. Foremost among these is the use of superhydrophobic surfaces for preventing ice formation $^{3-9}$. This concept draws inspiration from various natural materials, found in both animals and plants, that rely on a combination of surface morphology and surface chemistry to repel water ${ }^{10-14}$ from their surfaces. Figure 1 shows the result of the impact of water droplets on room temperature superhydrophobic surfaces; it is seen that the droplets completely bounce off the surface. We have recently reported a comprehensive experimental study that examined droplet impact on superhydrophobic surfaces and their ice-repelling properties under freezing conditions ${ }^{8}$. This work developed a new concept that the limited time of contact of impacting liquid on a superhydrophobic surface allows a supercooled water droplet to bounce off the surface before it can freeze (even at deep supercooling), thus fully preventing ice formation. Furthermore, it was $\operatorname{seen}^{8}$ that superhydrophobic surfaces completely prevent ice formation up to substrate temperatures as low as -20 to $-25^{\circ} \mathrm{C}$, whereas the hydrophilic and hydrophobic surfaces lead to significant ice accumulation since the droplets do not rebound completely. The existence of a critical substrate temperature ${ }^{8}$, above which ice formation from 
droplet impact (on superhydrophobic surfaces) is fully prevented has not been explored in any of the other existing studies of surface icing.

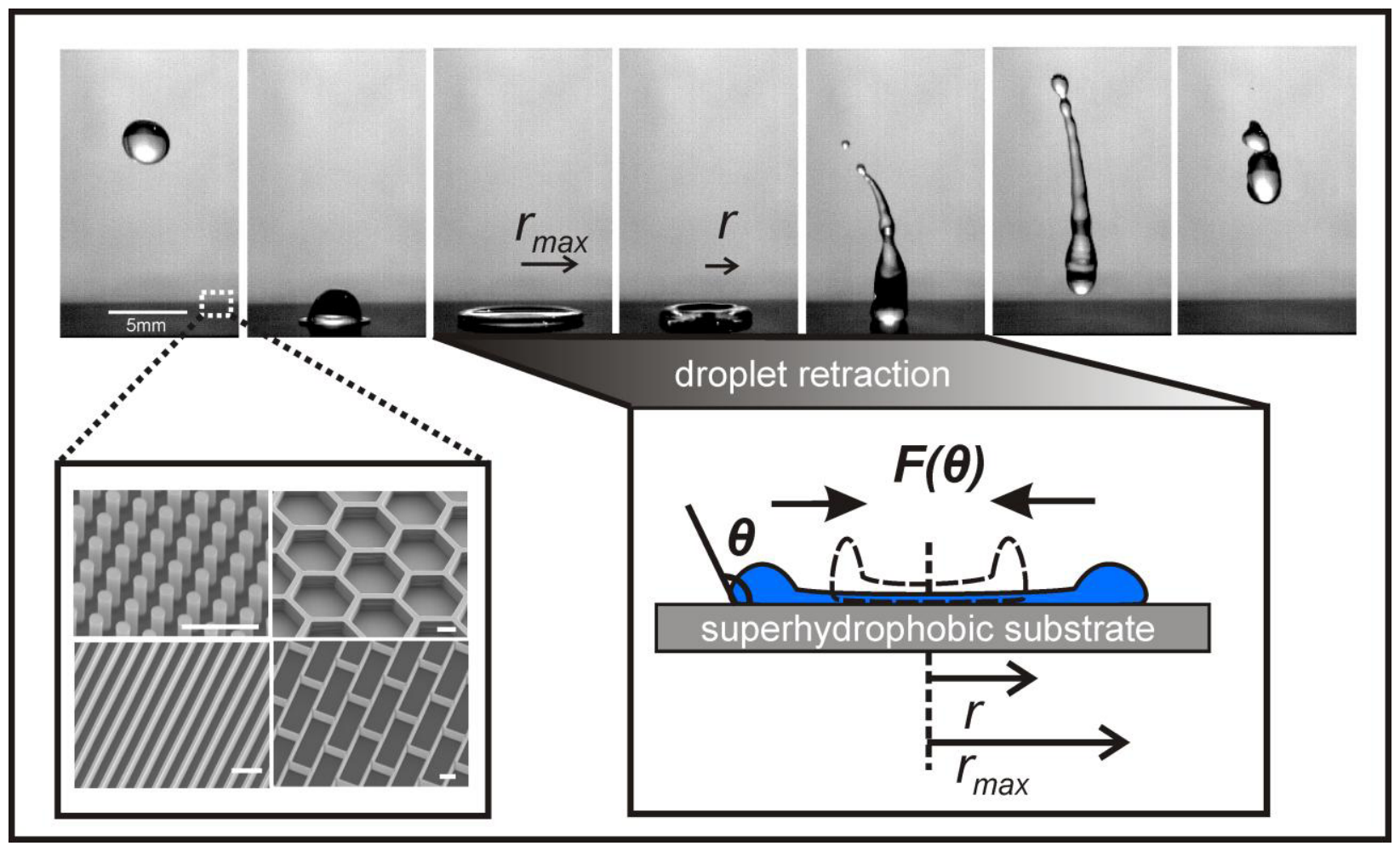

Figure 1: Impact behavior of $\sim 15 \mu L$ water droplets impacting a superhydrophobic surface. Images from left to right depict various stages of the droplet (released from a height of $10 \mathrm{~cm}$ ) spreading to a maximum radius $\left(r_{\max }\right)$, and then retracting completely from the surface. The four SEM images are illustrative superhydrophobic surfaces with posts, honeycombs, bricks and blade structures (scale bar: $10 \mu \mathrm{m})$. Also included is a schematic showing the droplet represented as a lumped toroidal mass during the spreading and retraction stage.

There have been other recent efforts to study superhydrophobic surfaces to control phase change phenomena of condensation ${ }^{15}$, boiling ${ }^{16}$ and freezing ${ }^{3-7,9}$. Most existing studies of ice accumulation on superhydrophobic surfaces have focused on the reduction in adhesion strength ${ }^{3,4}$ and delayed nucleation ${ }^{5-7}$. Cao et al. $^{7}$ demonstrated that nanostructured surfaces repel supercooled liquids and reduce ice accumulation, but there was marginal focus on studying ice repellency under liquid impact which is common in most icing situations. Studies of static droplets ${ }^{17-23}$ on superhydrophobic surfaces, and droplet impact dynamics ${ }^{24-26}$ on these surfaces have been conducted, but most studies are confined 
to room temperature and elevated temperature conditions. Another notable limitation of the existing reports is the absence of any theoretical approach to study the dynamic aspects of ice formation on surfaces. A theoretical study of dynamic ice formation on surfaces requires a multiphysics approach that will draw on the fields of fluid dynamics, heat transfer, surface chemistry, and nucleation theory.

The present work addresses this important gap in the existing body of literature and presents a predictive modeling framework for the study of dynamic ice formation on superhydrophobic surfaces; this model was utilized to explain the experimental observations in our previously reported work and is developed in detail in the present work ${ }^{8}$. The model is analytical in nature and only one empirical factor is used. The modeling framework consists of three submodels, which describe droplet impact dynamics, heat transfer and heterogeneous ice nucleation on superhydrophobic surfaces, respectively. These submodels are then integrated to predict the occurrence of ice formation upon impact of a supercooled droplet on a cold superhydrophobic surface. The predicted transition temperature for droplet freezing upon impact shows excellent agreement with the experimental data.

\section{Modeling ice formation on superhydrophobic surfaces}

This section presents details of the modeling framework used to predict ice formation on superhydrophobic surfaces. The modeling describes the phenomenon of a droplet impacting a superhydrophobic substrate, and the concurrent thermal and nucleation processes unfolding during the spreading and retraction stages of droplet impact. The superhydrophobic surface utilized in the present model consists of regularly arranged circular posts; however it is important to note that the modeling framework can be utilized for the analysis of ice formation on any superhydrophobic surface morphology. The modeling framework consists of three separate submodels, which predict droplet impact dynamics, heat transfer, and heterogeneous nucleation; the three submodels are described below. 


\section{2a. Droplet impact dynamics model}

This submodel predicts the spreading and retraction dynamics of a droplet impacting a superhydrophobic surface. In deriving the governing dynamics equation it is assumed that the mass of the droplet is localized near the peripheral contact line of the droplet as shown in Figure 1, in agreement with the experimentally observed toroidal shape of a droplet impacting superhydrophobic surfaces (see spreading image in Fig. 1). To a first order, the expansion and retraction processes can be modeled as a harmonic oscillator ${ }^{24}$, with the entire droplet represented as a lumped mass. Upon impact, the droplet tends to spread due to the inertia; this is countered by surface tension forces which act to prevent droplet spreading. The droplet spreads to a maximum radius, at which point surface tension forces cause it to begin retracting. The governing equation for a droplet retracting (or spreading) on a superhydrophobic surface can be established by balancing the horizontal forces at the contact line as:

$m_{0} \ddot{r}+2 \pi \gamma r(1-\cos \theta)=0$

where $m_{0}$ is the droplet mass, $r$ is the radial position of the droplet contact line, $\gamma$ is the liquid surface tension and $\theta$ is the macroscopic contact angle (advancing/retracting) of the droplet. The first term in the above equation represents droplet inertia and the second term represents the surface tension-based retraction force that acts radially inwards; the $2 \pi \gamma r(1-\cos \theta)$ term is the difference in the surface tension forces on the two sides of the toroidal droplet shown in Figure 1. It is important to highlight the absence of any friction term in the above equation; this is a consequence of the frictionless nature of the superhydrophobic surface. For a flat non-superhydrophobic surface the above equation can be modified to include another term corresponding to contact line friction. It also follows from the harmonic oscillator model that the spreading and retraction time of the droplet does not depend on the impact velocity ${ }^{24}$ since the spreading and retraction are governed only by the liquid surface tension. It should also be noted that the extent of the spreading depends on the droplet volume.

Equation 1 can be nondimensionalized as follows: 
$\frac{d^{2} R}{d t^{2}}+\frac{1}{2}(1-\cos \theta) R=0$

where $R=\frac{r}{r_{\max }}, t=\frac{t_{r}}{\tau}$ and $\tau=\sqrt{\frac{m_{0}}{4 \pi \gamma}}$.

In the above equations $R$ is dimensionless position of the contact line, $t$ is dimensionless retraction time $\left(t_{r}\right), \tau$ is the characteristic time constant and $r_{\max }$ is the maximum spread radius of the droplet. The second term in this equation represents the retraction force $F(\theta)$ that depends on the apparent receding contact angle $\theta$. It will be shown later in this section that the change in the receding contact angle resulting from ice nucleation reduces the retraction force responsible for dewetting.

\section{2b. Model to predict heat transfer on superhydrophobic surfaces}

During the time interval over which the droplet spreads and retracts, heat will be transferred from the relatively hot droplet to the substrate (for conditions where the substrate is at a lower temperature than the droplet). The thermal barrier for heat transfer to the substrate is provided by the posts and the trapped air layer underneath the droplet. We developed a simplified 1-D model to predict the transient temperature distribution inside the droplet while it is in contact with the superhydrophobic surface during spreading and retraction. It is important to note that this heat transfer model can be utilized for predicting the transient temperature distributions on any surface morphology and is not restricted to postlike geometries. The model neglects fluid convection effects due to the small size of the droplet. The temperature distribution in the droplet can be estimated by solving the heat conduction equations for two separate domains which are shown in Figure 2. The first domain is the droplet, while the second domain consists of the space occupied by the posts and the air beneath the droplet. The conduction equations for the post-air domain and the droplet are as follows:

$\frac{\partial T_{n s}\left(x, t_{c}\right)}{\partial t_{c}}=\alpha_{n s} \frac{\partial^{2} T_{n s}\left(x, t_{c}\right)}{\partial x^{2}}$ in the region $(0<x<h)$ 
$\frac{\partial T_{w}\left(x, t_{c}\right)}{\partial t_{c}}=\alpha_{w} \frac{\partial^{2} T_{w}(x, t)}{\partial x^{2}}$ in the region $(x>h)$

where $T_{n s}$ is the temperature of the post-air domain, $T_{w}$ is the droplet temperature, $h$ is the height of the posts, $t_{c}$ is the total time of contact of liquid at a particular radial position (spreading and retraction) and $\alpha_{n s}$ and $\alpha_{w}$ are the thermal diffusivities of the post-air domain (volume averaged properties of post material and air) and water respectively (see Supplementary for details). The two heat conduction equations are coupled through the common interface temperature at the droplet-substrate interface at $x=h$. The first boundary condition is based on the assumption that the substrate temperature $T_{\text {substrate }}$ is constant at all times; this assumption takes care of the initial and boundary condition at the substrate post-air domain interface. The second boundary condition follows from the fact that the droplet temperature at a position far away from the interface will equal the initial droplet temperature $T_{\text {droplet. }}$ The two coupled equations can be solved to get analytical expressions for the temperature distributions in the post-air domain and the droplet; these solutions are valid for all times and the detailed expressions are provided in the supplementary. The main parameter of interest, however, is the transient temperature variation at the droplet-substrate interface since ice nucleation will start at the interface. The transient temperature of the droplet-substrate interface $T_{\text {interface }}\left(t_{c}\right)$ can be estimated as (see supplementary for details):

$$
T_{\text {interface }}\left(t_{c}\right)=T_{\text {droplet }}+\left(T_{\text {substrate }}-T_{\text {droplet }}\right) \frac{\operatorname{erfc}\left(\frac{h}{2 \sqrt{\alpha_{n s} t_{c}}}\right)}{\operatorname{erfc}\left(\frac{h}{2 \sqrt{\alpha_{w} t_{c}}}\right)}
$$

where erfc denotes the complementary error function. 


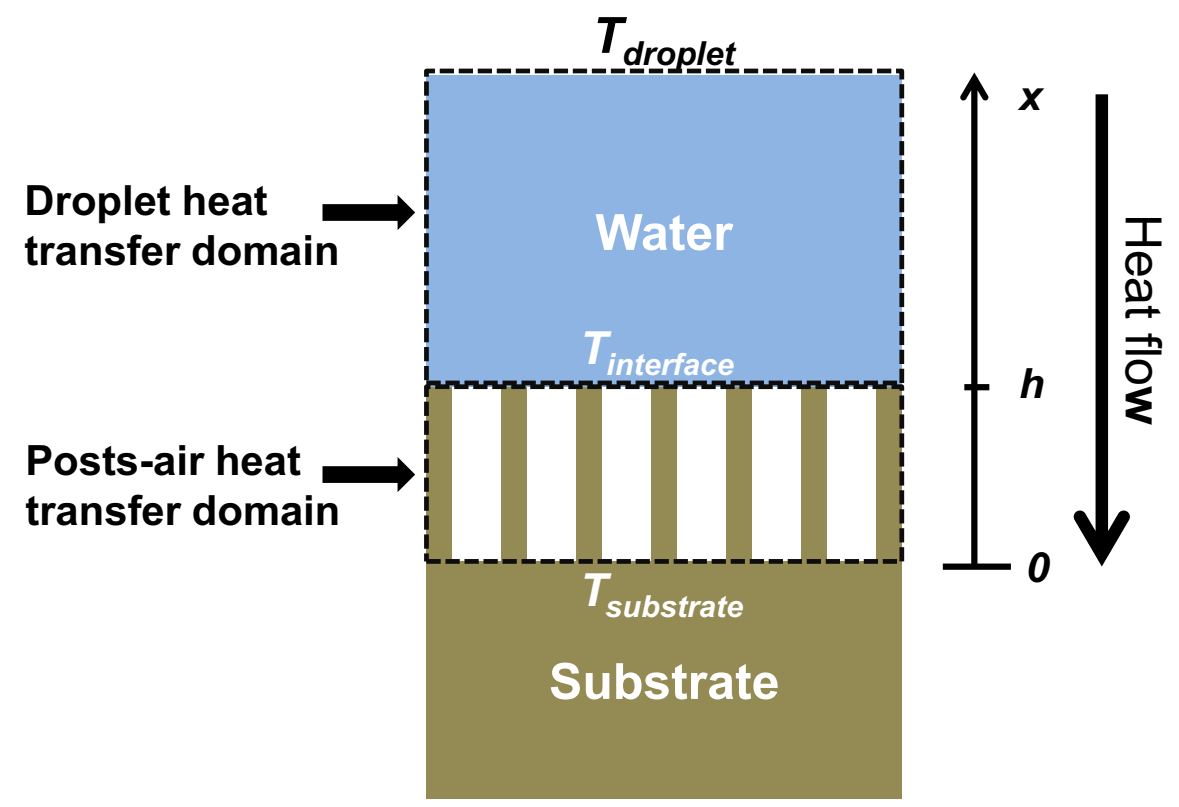

Figure 2: Model for predicting heat transfer from the droplet to the superhydrophobic surface, through the thermal resistance imposed by the posts and the air beneath the droplet (figure is not to scale)

\section{2c. Heterogeneous ice nucleation}

This submodel predicts the kinetics of nucleation of ice clusters on individual posts while the droplet is in contact during the spreading and retraction stages. Classical nucleation theory states that any nucleation event requires overcoming the nucleation free energy barrier. This free energy barrier is lowered when nucleation occurs on a foreign surface (heterogeneous nucleation). The free energy barrier $(\Delta G)$ associated with heterogeneous ice nucleation can be estimated $\operatorname{as}^{27}$ :

$\Delta G=\frac{16 \pi \gamma_{S L}^{3} T_{s l f}^{2}}{3 H_{S L}^{2}\left(T_{s l f}-T_{\text {interface }}\right)^{2}} S\left(\theta_{c}\right)$

where $s\left(\theta_{C}\right)=\frac{1}{2}+\frac{3}{4} \cos \theta_{C}-\frac{1}{4} \cos ^{3} \theta_{C}$

In the above equations $\gamma_{S L}$ is the solid-liquid (ice-water) interfacial energy, $T_{s l f}$ is the temperature of the freezing front, $H_{S L}$ is the latent heat of freezing and $\theta_{C}$ is the contact angle of ice on a flat surface in a water ambient. The parameter $s\left(\theta_{C}\right)$ accounts for the effect of heterogeneous nucleation and ranges between 0 and 1 with $s\left(\theta_{C}\right)=1$ corresponding to homogeneous nucleation conditions ${ }^{27}$. The value of $\theta_{C}$ 
has been assumed as $90^{\circ}$, based on the premise that the surface-ice and the surface-water interfacial energies are similar; furthermore a sensitivity analysis reveals that the results do not show a strong dependence on $\theta_{C}$.

The nucleation $\operatorname{rate}^{28} J\left(t_{c}\right)$ depends on the free energy barrier and can be expressed as:

$$
J\left(t_{c}\right)=K \exp \left(-\frac{\Delta G}{k T_{\text {interface }}}\right)
$$

where $K$ is a kinetic coefficient ${ }^{28}$ for nucleation and $k$ is the Boltzmann constant. It should be noted that the nucleation rate shows an exponential dependence on the free energy barrier and the temperature of the water-substrate interface $T_{\text {interface }}$.

\section{2d. Integrated model for predicting ice formation on superhydrophobic surfaces}

This section combines the three submodels described previously to develop a framework for predicting the supercooled conditions under which impacting droplets will either successfully retract and leave the surface, or pin and freeze in place. Firstly, the dynamics model is utilized to estimate the total time of contact of liquid with the surface as a function of the radial distance. The droplet dynamics equation (equation 2) can be solved using the harmonic oscillator approach; the results show that the total spreading time of the droplet is:

$$
t_{s}=\frac{\pi}{2} \tau \text {. }
$$

Furthermore, the time of contact of liquid with the surface (during spreading) as a function of the radial distance $R$ can be obtained from the solution of equation 2 as:

$t_{s}(R)=\tau\left(\frac{\pi}{2}-\sin ^{-1} R\right)$

It has been experimentally observed ${ }^{8}$ that pinning and freezing of the droplet occurs during the retraction stage. For convenience the time $t_{c}=0$ is therefore taken as the time corresponding to the 
maximum spread position of the droplet. The total contact time $\left(t_{c}\right)$ of the liquid at any radial position $r$ can be expressed (using equation 10) in terms of retraction time $t_{r}$ as:

$t_{c}=\tau\left(\frac{\pi}{2}-\sin ^{-1}(R)\right)+t_{r}$

During the expansion and retraction processes, ice will start nucleating at the tips of the posts. In this analysis it is assumed that the nucleating ice propagates as a hemispherical cap (as shown in Figure 3). This ice formation effectively increases the $\phi$-ratio (ratio of the top area of the roughness-causing features to the base area) and hydrophilicity of the surface while the droplet is spreading and retracting. The $\phi$-ratio variation depends on the total time of contact $\left(t_{c}\right)$ (spreading and retraction) and can be expressed using a Taylor series expansion as:

$\phi\left(t_{c}\right)=A_{1} t_{c}+A_{2} t_{c}^{2}+\ldots$

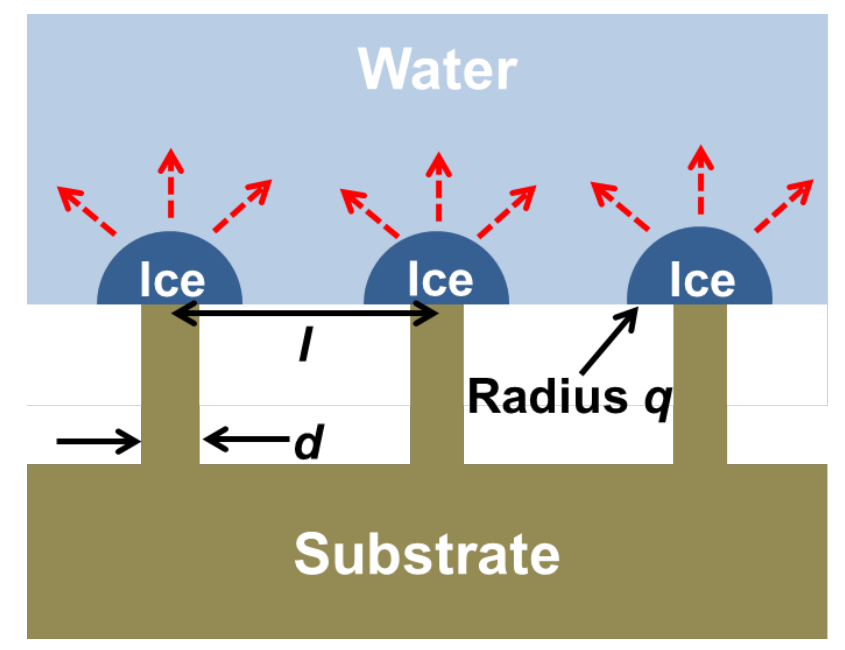

Figure 3: Schematic depicting the formation and propagation of nucleated ice as hemispherical caps on the posts. The increase in the size and hydrophilicity of the ice caps decreases the macroscopic contact angle of the droplet on the surface.

The increase in the $\phi$-ratio and the change in surface chemistry due to the formation of an ice layer result in a change in the macroscopic receding contact angle of the droplet. The contact angle of a droplet in the Cassie state is predicted by the Cassie equation ${ }^{29}$ as: 
$\cos \theta=-1+\phi\left(1+\cos \theta_{f}\right)$

where $\theta_{f}$ is the contact angle of the droplet on a flat surface of the same material as that of the posts.

In the present situation, the water droplet spreads and retracts on the nucleated ice layer; the contact angle of water on ice is assumed to be zero, i.e. water wets ice perfectly. Equation 13 for the macroscopic contact angle thus reduces to:

$\cos \theta=-1+2 \phi$

Equations 12 and 14 can be incorporated in the droplet dynamics model (equation 2) which yields:

$\frac{d^{2} R}{d t^{2}}+\left(1-A_{1} t_{c}-A_{2} t_{c}^{2}+\ldots.\right) R=0$

where $t$ is again dimensionless retraction time.

The time of contact $t_{c}$ can be nondimensionalized as $t_{c} / X_{c}=\xi$, where $X_{c}=\tau\left(\frac{\pi}{2}-\sin ^{-1}(R)\right)+t_{r}$, which results in:

$\frac{d^{2} R}{d t^{2}}+\left(1-B_{1} X_{c}-B_{2} X_{c}^{2}+\ldots.\right) R=0$

where $B_{1}=A_{1} \xi$ and $B_{2}=A_{2} \xi^{2}$.

The second term in equation 16 is the retraction force on the droplet $F(\theta)$, as a function of the dimensionless contact time $X_{c}$. The solution of equation 16 predicts the location of the droplet contact line $(R)$ with time.

The constants $B_{1}$ and $B_{2}$ can be estimated by relating the Taylor series expansion of the $\phi$-ratio (equation 12) to the $\phi$-ratio obtained using heterogeneous nucleation theory and freeze-front propagation rates. It is assumed that ice nucleates and propagates as hemispherical clusters as shown in Figure 3; the cumulative transient $\phi$-ratio of a surface consisting of posts with such expanding ice caps (radius $q$ ) can be expressed as: 
$\phi\left(t_{c}\right)=2 \pi \cdot \phi_{0} \int_{0}^{t_{c}} J(\hat{t})\left[q \cdot\left(t_{c}-\hat{t}\right)\right]^{2} d \hat{t}$

where $J$ is the nucleation rate, $\phi_{0}$ is the $\phi$-ratio of the surface without any ice accumulation, and $q$ is the radius of the ice cap. It should be noted that the area of ice which contacts the droplets (and determines the $\phi$-ratio) is the hemispherical curved surface area, and not the base area of the hemisphere. The initial $\phi$-ratio $\phi_{0}$ of the surface considered in this study (circular posts of diameter $d$ with square pitch $l$ ) can be expressed as:

$\phi_{0}=\frac{\pi d^{2}}{4 l^{2}}$

The ice caps will grow larger with time as the freeze front propagates. For a one-dimensional ice growth approximation, the freeze-front propagation rate can be expressed $\mathrm{as}^{30}$ :

$q\left(t_{c}-\hat{t}\right)=U\left(T_{\text {sub }}, T_{\mathrm{droplet}}, T_{s l f}\right) \sqrt{\left(t_{c}-\hat{t}\right)}$

where

$U=\left(4 \alpha_{i c e} \frac{C_{p_{-} i c e}}{2 H_{S L}}\left(T_{s l f}-T_{s u b}\right)\left[1+\frac{k_{w}}{k_{i c e}} \sqrt{\frac{\alpha_{w}}{\alpha_{i c e}}} \frac{\left(T_{s l f}-T_{\mathrm{drplec}}\right)}{\left(T_{s l f}-T_{\text {sub }}\right)}\right]\right)^{\frac{1}{2}}$

is a measure of the speed of propagation of the freeze-front. Equations 8 and 17-20 can be used to estimate the transient $\phi$-ratio due to ice formation as:

$\phi\left(t_{c}\right)=2 \pi \phi_{0} K U^{2} \int_{0}^{t_{c}} \exp \left(-\frac{\Delta G}{k T(\hat{t})}\right)\left(t_{c}-\hat{t}\right) d \hat{t}$

Expansion of the above equation using a Taylor series and comparison with equation 12 results in the values of the constants we need in Equation 16:

$$
\begin{aligned}
& B_{1}=0 \\
& A_{2}=\pi \cdot \phi_{0} K U^{2} \exp \left(-\frac{\Delta G}{k T_{\text {intef face }}}\right) B_{2}=\tau^{2} \pi \cdot \phi_{0} K U^{2} \exp \left(-\frac{\Delta G}{k T_{\text {interface }}}\right)
\end{aligned}
$$


The equation governing the dynamics of droplet impact on supercooled superhydrophobic surfaces can now be written using Equation 16 as:

$$
\frac{d^{2} R}{d t^{2}}+\left(1-C \cdot \phi_{0} \cdot e^{f\left(T_{\text {inerface }}\right)} \cdot t_{c}^{2}\right) R=0
$$

where $C$ combines various parameters in equation 23 and can be represented as:

$$
C=\pi K U^{2}
$$

The function $f\left(T_{\text {interface }}\right)$ highlights the significant influence of the nucleation free energy barrier (that depends on the interface temperature) on the droplet dynamics and the freezing process. $f\left(T_{\text {interface }}\right)$ can be represented using equations 6 and 8 as:

$$
f\left(T_{\text {interface }}\right)=-\frac{16 \pi \gamma_{S L}^{3} T_{s l f}^{2}}{3 k H_{S L}^{2}\left(T_{s l f}-T_{\text {interface }}\right)^{2} T_{\text {interface }}} s\left(\theta_{C}\right)
$$

Furthermore, the dependence of $T_{\text {interface }}$ on $T_{\text {substrate }}$ and $T_{\text {droplet }}$ can be obtained using equation 5.

Equation 24 can be solved numerically and yields the droplet retraction position versus time; this information can be utilized to predict the occurrence of freezing as detailed in the next section.

\section{Modeling results and comparison with experiments}

The equations developed in the previous section can be used to predict the conditions that would result in complete retraction or pinning and freezing of a droplet impacting a superhydrophobic surface. The key to the prediction of ice formation is equation 24; the solution of this equation yields the evolution of the retraction $\operatorname{position}(R)$ of the droplet contact line as a function of time. It should also be noted that the second term in equation 24 is the time-dependent retraction force acting on the droplet; this retraction force decreases with time under freezing conditions. A comparison of the profiles of the droplet contact line position and the retraction force can be used to predict the occurrence of freezing. If the retraction force is positive over the entire time interval of droplet retraction, the droplet will dewet completely and there will be no ice formation. If however, the retraction force becomes zero or 
negative before the droplet contracts fully, the droplet will pin at that location and eventually freeze. Thus the central concept for avoiding ice formation upon liquid impact is surface design and conditions which ensure a positive retraction force throughout the retraction stage.

To verify the models developed in this work, carefully planned experiments were conducted to study freezing upon droplet impact on superhydrophobic surfaces. ${ }^{8}$ In this study, $2.5 \mathrm{~mm}$ diameter water droplets at $20^{\circ} \mathrm{C}, 60^{\circ} \mathrm{C}, 0^{\circ} \mathrm{C}$ or supercooled to $-5^{\circ} \mathrm{C}$, were impacted onto superhydrophobic surfaces at controlled substrate temperatures $\left(-35^{\circ} \mathrm{C}\right.$ to $\left.20^{\circ} \mathrm{C}\right)$. The water droplets were released from a height of 10 $\mathrm{cm}$ and impacted the surfaces at a $90^{\circ}$ angle. A simple heat transfer analysis indicates that the droplet temperature will not change substantially during the time of flight. The whole experimental apparatus was enclosed in a custom made environmental chamber that was maintained at room temperature and 5 $\%$ relative humidity. Droplet impact dynamics were studied by utilizing a high-speed camera to record the spreading and retraction stages of the droplet. The surfaces selected for the present experiments consisted of silicon posts that were silanized to impart superhydrophobicity. The circular posts had a diameter of $1.5 \mu \mathrm{m}$, a square pitch of $3.6 \mu \mathrm{m}$ and a height of $10 \mu \mathrm{m}$ (the $\phi$-ratio $\phi_{0}$ of the surface is 0.14 ). More details about the experimental apparatus and the methods employed for surface fabrication are provided in the recent work by Mishchenko et al. ${ }^{8}$

The predictive capabilities of the presently developed modeling framework are demonstrated in Figures $4 \mathrm{a}-\mathrm{c}$ that show the dimensionless droplet contact line radius and the retraction force as a function of the retraction time for three different substrate temperatures (the initial droplet temperature is $20^{\circ} \mathrm{C}$ ). At the substrate temperature corresponding to Figure $4 \mathrm{a}$, it is seen that the retraction force is positive throughout the retraction time interval (i.e until the radius becomes zero); the existence of a positive force implies that the droplet will successfully bounce off without freezing on the surface. This is demonstrated schematically in Figure $4 \mathrm{a}$ and matches the experimental observations ${ }^{8}$ wherein the droplet indeed completely comes off the surface after impact. Figure $4 \mathrm{~b}$ corresponds to a lower substrate temperature; the plots show that the retraction force becomes zero at the same instant that the droplet radius becomes zero. This is the critical substrate temperature at which the droplet will barely 
come off the surface; this is schematically shown in Figure $4 \mathrm{~b}$ and again matches the experimental results $^{8}$. Figure $5 \mathrm{c}$ corresponds to even lower substrate temperatures; it is seen that the retraction force becomes zero before the droplet can fully retract. This will inhibit the ability of the droplet to bounce off; it will pin at that location and subsequently freeze as is experimentally observed ${ }^{8}$.

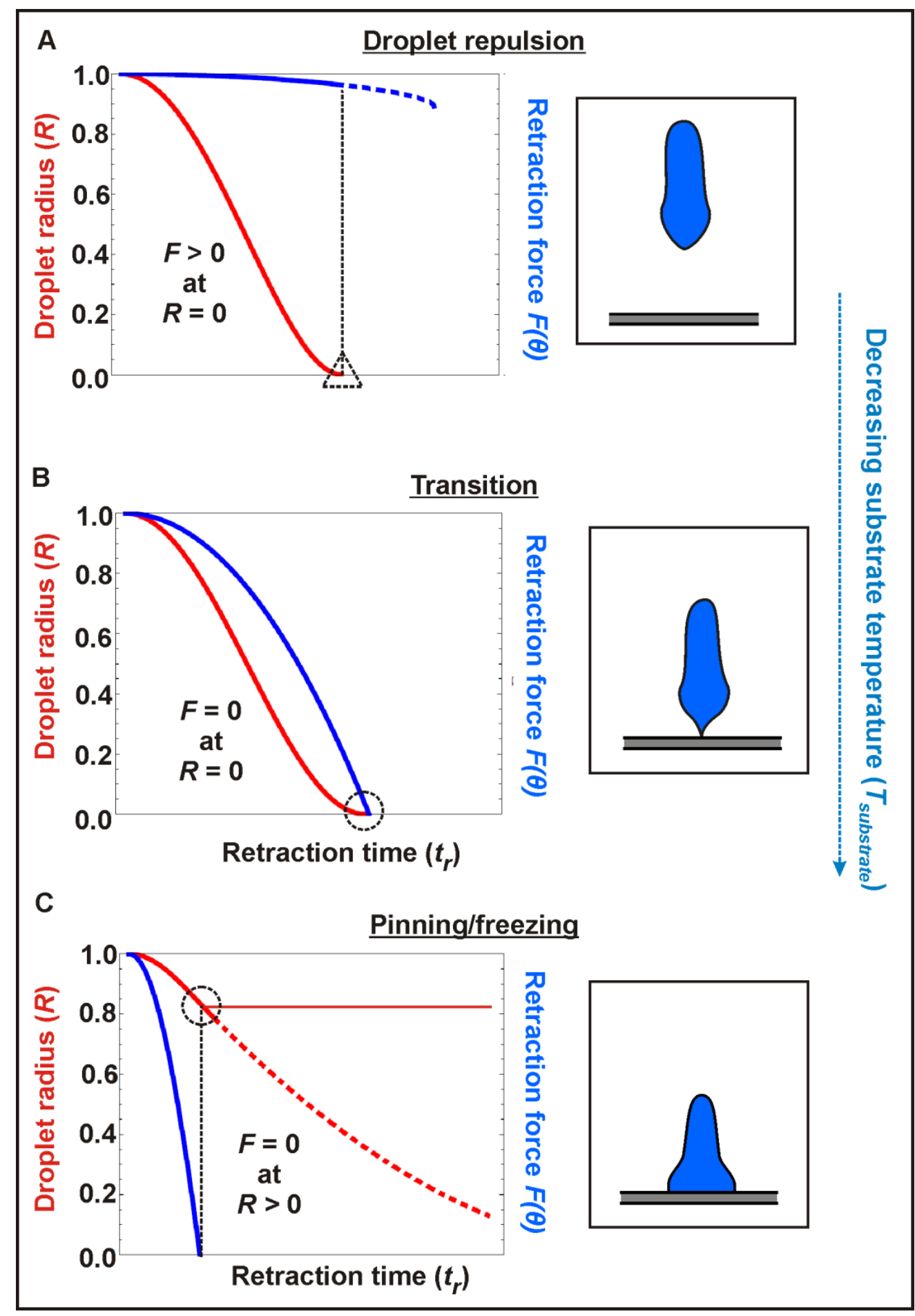

Figure 4. Plots of dimensionless radius and retraction force versus retraction time for three different substrate temperatures; the schematics on the right show the results of droplet impact. a.) Complete 
bounce back of the droplet after impact; this occurs because the retraction force is positive throughout the retraction time interval, b.) Critical substrate temperature at which the droplet just comes off the surface; this occurs because the retraction force becomes zero at $R=0$, and c.) Droplet freezes during retraction; this corresponds to the retraction force becoming zero before the droplet can fully retract.

The model thus clearly predicts the existence of a critical substrate temperature below which liquid droplets will freeze upon impact; no ice formation is expected above this critical substrate temperature. This critical temperature depends on surface morphology, surface chemistry and the thermal properties of the substrate and the liquid. For the present set of experiments, the model predicts that this critical transition temperature of the substrate is $\sim-20{ }^{\circ} \mathrm{C}$ (see Supplementary for details). This is in excellent agreement with our previous experimental results ${ }^{8}$, in which droplets freeze upon impact at substrate temperatures below $-20{ }^{\circ} \mathrm{C}$ to $-25^{\circ} \mathrm{C}$. It should also be noted that the model predicts the conservative (higher) estimate of the critical substrate temperature. In reality, ice formation is initiated after a finite induction time for nucleation elapses; this implies that the critical substrate temperature for freezing should be lower than the predicted value, which matches experimental observations.

The model can be utilized to study the influence of various surface parameters and material properties on the occurrence of freezing. An analysis of equation 24 shows that the retraction force is a strong function of the interface temperature $T_{\text {interface }}$; this is expected since the interface temperature determines the nucleation rate and the extent of propagation of the freeze front. The interface temperature is, in turn, determined by the initial droplet temperature $T_{\text {droplet }}$ and the substrate temperature $T_{\text {substrate }}$ as predicted by equation 5 . An examination of equation 5 reveals that the interface temperature is dominated by the substrate temperature and shows a weak dependence on the initial droplet temperature. This trend has clearly been noticed in the experimental results; the substrate temperature, at which a drop freezes upon impact, does not change significantly with initial droplet temperatures. It should be noted that this dominance of the substrate temperature $T_{\text {substrate }}$ in determining the interface temperature holds true only for silicon-based surfaces and is a consequence of the higher thermal diffusivity of silicon $\left(8.9 \times 10^{-5} \mathrm{~m}^{2} / \mathrm{s}\right)$ than water $\left(1.3 \times 10^{-7} \mathrm{~m}^{2} / \mathrm{s}\right)$. For the impact of 
water droplets on polymeric superhydrophobic surfaces (eg. PDMS with a thermal diffusivity of $1.08 \mathrm{x}$ $\left.10^{-7} \mathrm{~m}^{2} / \mathrm{s}\right)$, the contributions of the substrate and the initial droplet temperatures in determining $T_{\text {interface }}$ are expected to be comparable.

Control of the interface temperature $T_{\text {interface }}$ is an important tool to limit ice formation on superhydrophobic surfaces. Equation 5 also reveals the role of the superhydrophobic structure in influencing heat transfer and hence the subsequent freezing process. Equation 5 indicates a weak dependence of $T_{\text {interface }}$ on the height of posts; making the posts shorter or taller will thus not change the interface temperature significantly for a specified substrate and initial droplet temperature. Equation 24 also shows that the $\phi$-ratio of the posts $\left(\phi_{0}\right)$ appears outside the exponential term; this implies that the $\phi$ ratio will not significantly influence droplet freezing. However the $\phi$-ratio influences droplet dynamics; increasing the $\phi$-ratio reduces superhydrophobicity which will influence the droplet dynamics unfavorably and could lead to undesired pinning effects. Lastly, because the thermal diffusivities of air $\left(2.2 \times 10^{-5} \mathrm{~m}^{2} / \mathrm{s}\right)$ and silicon $\left(8.9 \times 10^{-5} \mathrm{~m}^{2} / \mathrm{s}\right)$ are of a similar magnitude, structuring the surface will not increase the transient heat transfer resistance significantly (despite the lower thermal conductivity of air as compared to silicon). Increasing the transient resistance to heat transfer from the droplet to the substrate is key to increasing $T_{\text {interface }}$ and reducing the probability of nucleation. Based on these observations, polymeric substrates are expected to have more favorable anti-icing properties because of their lower thermal diffusivities.

The above discussion illustrates the use of the present model to predict the influence of various parameters related to surface morphology, surface chemistry and thermal properties on dynamic ice formation on superhydrophobic surfaces. A key aspect of the modeling approach is the minimal reliance on empirical (one parameter) and measured data. The model can thus be easily extended and applied for analysis of freezing on other types of structures (eg. closed cell structures, hierarchical structures). To the best of the authors' knowledge, this model is the first detailed physics-based framework for the design of advanced surfaces for anti-icing applications. 


\section{Conclusions}

The present work developed a predictive modeling framework for understanding ice formation on superhydrophobic surfaces upon droplet-surface impact under freezing conditions. It is important to stress that the modeling is physics-based and reliance on empirical parameters is minimal (one parameter). The influence of surface chemistry, surface morphology, thermal properties and temperature gradients on ice formation can be clearly outlined. The model can be used to identify dominant parameters and conditions (from a vast parameter space) that will reduce ice formation. This work thus outlines the underlying principles that are critical in the design of advanced surfaces and strategies for mitigating ice formation. It is also important to note that the models developed in the present work have applications beyond ice formation and can be utilized for the study of other phasechange phenomena on superhydrophobic surfaces, such as boiling, condensation and sublimation.

\section{Nomenclature}

$A_{1}, A_{2}$ Taylor series coefficients for the expansion of the $\phi$-ratio

$B_{1}, B_{2}$ Taylor series coefficients for the expansion of the $\phi$-ratio (with the contact time nondimensionalized)

C constant which combines various parameters in the droplet dynamics equation

$C_{p_{-} i c e}$ specific heat capacity of ice

d diameter of posts

$F(\theta) \quad$ retraction force on the droplet

$h \quad$ height of posts

$H_{S L} \quad$ latent heat of freezing of ice

$k \quad$ Boltzmann constant

$k_{\text {ice }} \quad$ thermal conductivity of ice

$k_{w} \quad$ thermal conductivity of water 
$K \quad$ kinetic coefficient corresponding to freezing $\left(10^{24} \mathrm{~m}^{-2} \mathrm{~s}^{-1}\right)$

$l \quad$ pitch between posts

$m_{0} \quad$ droplet mass

$J \quad$ ice nucleation rate

$r \quad$ radial position of the droplet contact line

$r_{\max }$ maximum spread radius of the droplet

$R_{m} \quad$ maximum spread radius of the droplet

$q \quad$ radius of the hemispherical ice cap

$t \quad$ dimensionless retraction time of droplet

$t_{c} \quad$ total time of contact of liquid with substrate (at a particular droplet position)

$t_{r} \quad$ retraction time of droplet

$t_{s} \quad$ spreading time of droplet

$s \quad$ function which depends on the droplet-substrate interface temperature

$T_{s l f} \quad$ temperature of the freezing front $\left(0^{\circ} \mathrm{C}\right)$

$T_{\text {substrate }}$ temperature at the base of the structured substrate

$T_{d r o p l e t}$ temperature of the droplet before impacting the cold substrate

$T_{\text {interface }}$ transient temperature of the droplet-structured substrate interface

$T_{\text {sub_crit }}$ minimum temperature of the substrate at which water droplets pin upon impact

$U$ measure of the speed of propagation of the freezing front

Q $\alpha_{\text {ice }}$ thermal diffusivity of ice

$\alpha_{\mathrm{ns}} \quad$ thermal diffusivity of the structured substrate

$\alpha_{w} \quad$ thermal diffusivity of water

$\Delta G \quad$ free energy barrier for ice nucleation

$\gamma \quad$ surface tension (liquid-air)

$\gamma_{S L} \quad$ ice-water interfacial energy $(20 \mathrm{mN} / \mathrm{m})$

$\varnothing \quad \phi$-ratio of surface undergoing ice nucleation 
$\phi_{0} \quad$ the initial $\phi$-ratio of the surface (without any ice formation)

$\tau \quad$ time constant for droplet spreading and retraction

$\theta \quad$ macroscopic contact angle of droplet on superhydrophobic surface

$\theta_{C} \quad$ ice-flat surface contact angle $\left(90^{\circ}\right)$ in a water ambient

$\theta_{f} \quad$ contact angle of water on flat surface in air

\section{Acknowledgements}

This work was funded in part by DARPA (award number HR0011-08-C-0114). Lidiya Mischchenko thanks the U.S. Department of Homeland Security (DHS) Scholarship and Fellowship Program for financial support.

\section{References}

(1) Gent, R. W.; Dart, N. P.; Cansdale, J. T. Philosophical Transactions of the Royal Society A, 2000, 358, 2873.

(2) Dalili, N., Edrisy, A., Carriveau, R. Renewable and Sustainable Energy Reviews, 2009, $13,428$.

(3) Kulinich, S.A., Farzaneh, M. Langmuir, 2009, 25, 8854.

(4) Sarkar, D.K, Farzaneh, M. Journal of Adhesion Science and Technology, 2009, 23, 1215.

(5) Tourkine, P.; Le Merrer, M.; Quere, D. Langmuir, 2009, 25, 7214.

(6) He, M.; Wang, J. X.; Li, H. L.; Jin, X. L.; Wang, J. J.; Liu, B. Q.; Song, Y. L. Soft Matter, 2010, $6,2396$.

(7) Cao, L. L.; Jones, A. K.; Sikka, V. K.; Wu, J. Z.; Gao, D. Langmuir, 2009, 25, 12444.

(8) Mishchenko, L.; Hatton, B.; Bahadur, V., Taylor, J. A.; Krupenkin, T., Aizenberg, J. ACS Nano, 2010, 4, 7699.

(9) Kulinich, S. A.; Farhadi, S.; Nose, K. and Du, X. W. Langmuir, 2010, in press.

(10) Gao, X. F.; Yan, X.; Yao, X.; Xu, L.; Zhang, K.; Zhang, J. H.; Yang, B.; Jiang, L. Advanced Materials, 2007, 19, 2213. 
(11) Gao, X. F.; Jiang, L. Nature, 2004, 432, 36.

(12) Autumn, K.; Liang, Y. A.; Hsieh, S. T.; Zesch, W.; Chan, W. P.; Kenny, T. W.; Fearing, R.; Full, R. J. Nature, 2000, 405, 681.

(13) Zheng, Y. M.; Gao, X. F.; Jiang, L. Soft Matter, 2007, 3, 178.

(14) Patankar, N. A. Langmuir, 2004, 20, 8209.

(15) Varanasi, K. K.; Hsu, M.; Bhate, N.; Yang, W. S.; Deng, T. Applied Physics Letters, 2009, 95, 094101.

(16) Patankar P.; Soft Matter, 2010, 6, 1613.

(17) Krupenkin, T. N.; Taylor, J. A.; Wang, E. N.; Kolodner, P.; Hodes, M.; Salamon, T. R. Langmuir, 2007, 23, 9128.

(18) Ahuja, A.; Taylor, J. A.; Lifton, V.; Sidorenko, A. A.; Salamon, T. R.; Lobaton, E. J.; Kolodner, P.; Krupenkin, T. N. Langmuir, 2008, 24, 9.

(19) Tuteja, A.; Choi, W.; Ma, M. L.; Mabry, J. M.; Mazzella, S. A.; Rutledge, G. C.; McKinley, G. H.; Cohen, R. E. Science, 2007, 318, 1618.

(20) Roach, P.; Shirtcliffe, N. J.; Newton, M. I. Soft Matter, 2008, 4, 224.

(21) Li, X. M.; Reinhoudt, D.; Crego-Calama, M. Chemical Socity Reviews, 2007, 36, 1350.

(22) Patankar, N. A. Langmuir, 2004, 20, 7097.

(23) Bahadur, V.; Garimella, S. V. Langmuir, 2009, 25, 4815.

(24) Richard, D.; Clanet, C.; Quere, D. Nature, 2002, 417, 811.

(25) Deng, T.; Varanasi, K. K.; Hsu, M.; Bhate, N.; Keimel, C.; Stein, J.; Blohm, M. Applied Physics Letters, 2009, 94, 133109.

(26) Jung, Y. C.; Bhushan, B. Langmuir, 2008, 24, 6262.

(27) Zettlemoyer, A. C. Nucleation, M. Dekker, 1969.

(28) Fletcher, N. H. Journal of the Atmospheric Sciences, 1969, 26, 1266.

(29) Cassie, A. B. D. Discussions of the Faraday Society, 1948, 3, 11. 
(30) Poulikakos, D. Conduction Heat Transfer, Prentice Hall, 1994.

\section{Table of Contents Graphic}

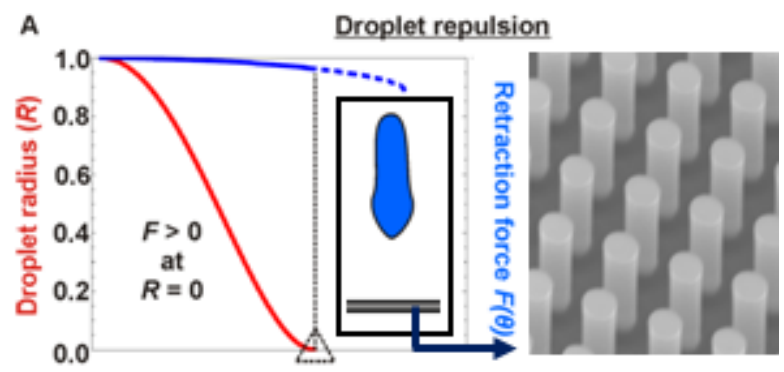

